\title{
Investigation of a New Idea for Antipodal Vivaldi Antenna Design
}

\author{
Mohammad Hamed Habib Agahi, Habibollah Abiri and Farzad Mohajeri
}

\begin{abstract}
A novel method for improving the performance of Antipodal Vivaldi antenna is proposed and verified for its effectiveness. An Antipodal Vivaldi antenna is an UWB (Ultra Wide Band) antenna; however, its VSWR can be improved by employing a new procedure. Accordingly, a parasitic parameter is inserted in the structure in order to make the current distribution smoother. The reflection coefficient, radiation patterns and current distribution for both structures (the common and modified Antipodal Vivaldi antennas) are analyzed and compared, using full wave simulation tools.
\end{abstract}

Index Terms-Antipodal Vivaldi antenna, current distribution, parasitic element, ultra wide band.

\section{INTRODUCTION}

Vivaldi type antennas have interesting performances compared to other wide band antennas considering their multi-octave bandwidth and wide angle scan capabilities of their arrays. That is why Vivaldi antennas can be used in different wideband electronic systems such as satellite communications, electronic warfare systems, remote sensing and radio telescopes. These arrays are relatively easy to fabricate by using printed circuit techniques. Depending on the applications, feeding techniques prove to be convenient by manipulating microstrip or strip lines.

Various forms of Vivaldi antennas have been developed so far. However, the exponentially tapered slot antenna is the most popular solution. When adjacent elements are electrically connected along E-plane, the exponentially tapered slot antenna can provide a broad band (up to a ratio 4.5:1) within an array featuring a scan angle up to $45^{\circ}$ [1]. The transition from microstrip to slotline, however, restricts further extension of the impedance bandwidth. Furthermore, asymmetric feed structures tend to produce high crosspolarization. Antipodal Vivaldi antennas have been proposed as a solution for this problem. They utilize a smoothly tapered transition from microstrip to Antipodal slotline, which eliminates the bandwidth limitations of original Vivaldi antenna design [2]. In addition, recent developments on Antipodal Vivaldi array further illustrate that they can be formed as a modular construction of an array without the resonance anomalies that are present in classic Vivaldi array with gaps between elements [3]. The modular structure makes the fabrication and maintenance of the array easy. Therefore, Antipodal antenna offers more possibilities as a solution for a broadband array with

Manuscript received September 25, 2010

Department of Communication and Electronic Engineering, Shiraz University, Shiraz, Iran versatile usage.

Extensive studies have been carried out on Vivaldi antennas and their applications in arrays. However, due to the complexity of operating modes in wideband antennas, a routine design technique for Vivaldi antennas is not available, yet. It is difficult to determine the necessary changes that might lead to the improvement of the performance of a particular design.

Antipodal form of these antennas also faces a similar challenge.

It will be shown that by adding a parasitic element to the Antipodal Vivaldi antenna, a degree of freedom is obtained to improve the design. CST Microwave Studio software is used to simulate and compare the performance.

\section{DESIGN}

An Antipodal Vivaldi antenna has 3 basic parts: 1 . Transmission line, 2. Radiator, 3. Transition between transmission line and the radiator. The transmission line is a microstrip that is compatible with MMICs. Generally, the transition between transmission line and the radiator has an exponential form. Actually, this is the most important part that affects the impedance matching. These three parts can be described by appealing some parameters, introduced in [1]. The common structure is shown in Fig. 1. The dimension of the structure is $50 \times 25 \times 0.6 \mathrm{~mm}$. The objective was not optimizing the basic parameters; therefore, a small antenna was selected and the value of these parameters for a lower frequency band of $f_{L}=9 \mathrm{GHz}$ were approximated according to [1], [4] and presented in table 1 . The substrate thickness is $0.6 \mathrm{~mm}$, and the relative dielectric constant $\left(\varepsilon_{\mathrm{r}}\right)$ corresponding to Rogers RO3003, is 3.

This structure is simulated by CST Microwave Studio CAD tools. $\left|\mathrm{S}_{11}\right|$ and the antenna gain versus frequency are plotted in Fig. 2. It is seen that $\left|\mathrm{S}_{11}\right|$ is less than $-10 \mathrm{~dB}$ above $9.13 \mathrm{GHz}$, while the gain is more than $4 \mathrm{~dB}$ above 9 $\mathrm{GHz}$. The gain increases gradually and reaches approximately $9 \mathrm{~dB}$ at $20 \mathrm{GHz}$.

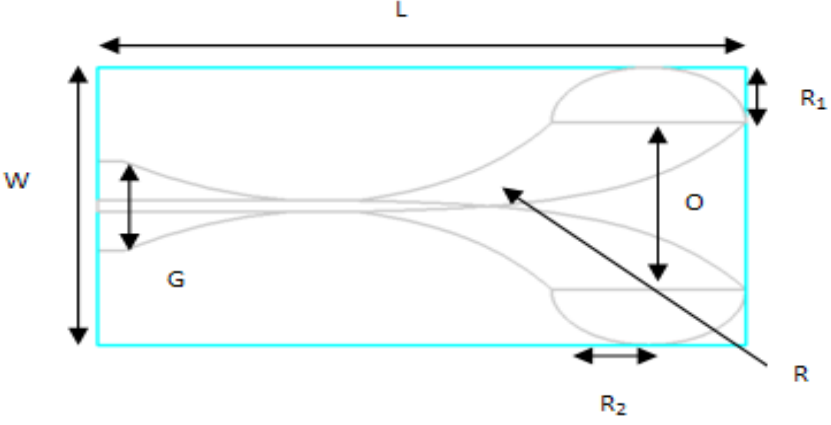

Fig. 1. Structure of common Antipodal Vivaldi antenna. 
TABLE 1. THE VALUES OF PARAMETERS DEFINED IN FIG.1.

\begin{tabular}{|c|c|c|c|c|c|c|}
\hline Parameter & $\mathrm{L}$ & $\mathrm{W}$ & $\mathrm{O}$ & $\mathrm{G}$ & $\mathrm{R}_{1}$ & $\mathrm{R}_{2}$ \\
\hline Size in mm & 50 & 25 & 15 & 8 & 5 & 7.5 \\
\hline \multicolumn{7}{|c|}{$\mathrm{R}$ is the power coefficient of the exponential term and is } \\
chosen to be 0.125.
\end{tabular}

\section{MODIFIED ANTIPODAL VIVALDI ANTENNA}

The opening rate $(\mathrm{R})$ determines the edge curve along which the current mainly concentrates, so it must be a critical parameter for antenna design [1]. As the opening rate increases, the lower end of frequency band decreases. However, the gain decreases as well. Hence, there is a tradeoff between bandwidth and antenna gain [1]. The objective is to add a new parameter to the structure in order to obtain a degree of freedom on $\mathrm{R}$, so that the lower end of frequency band decreases, whereas the gain be nearly the same or more than that of the common structure. The parasitic element is investigated by simulation. Monitoring the current distribution is the key to find the final element shape. The final element shape becomes an elliptical strip inserted adjacent to the common structure which makes the current distribution smoother. The modified structure is shown in Fig. 3 and the current distribution of both structures (common and modified Antipodal Vivaldi antennas) at $10.5 \mathrm{GHz}$ are shown in Fig. 4.

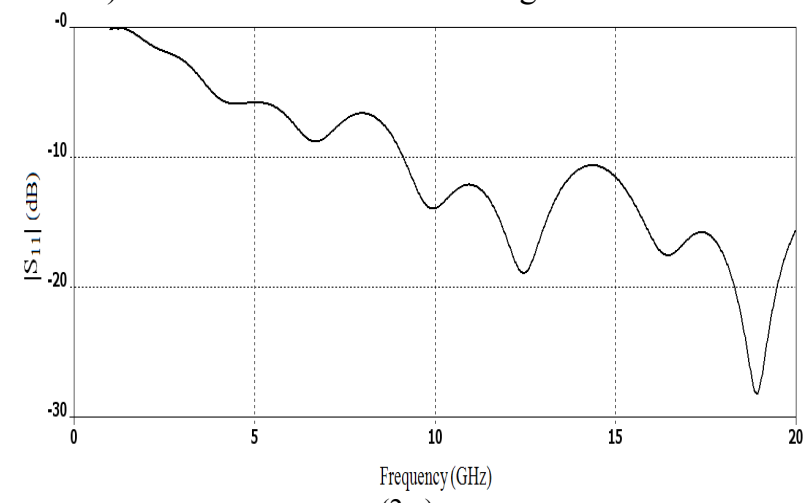

(2-a)

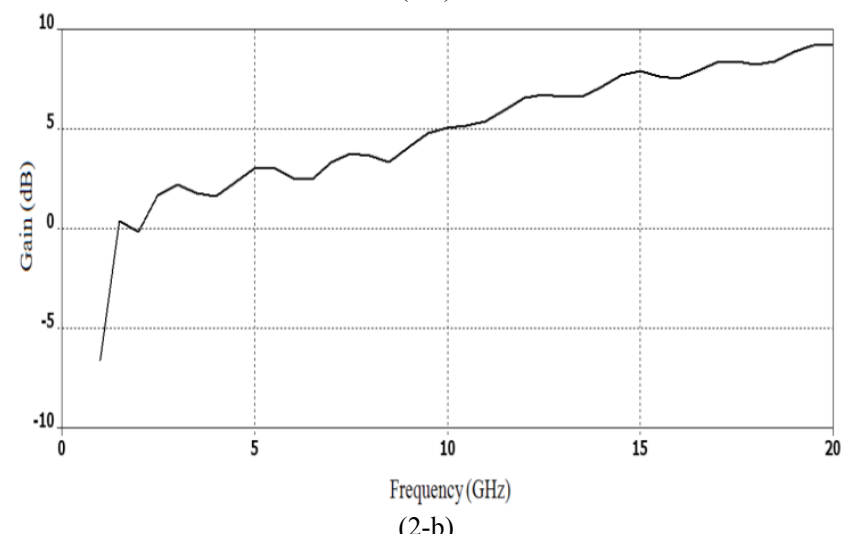

Fig. 2. a) $\left|S_{11}\right|$ and b) the gain of Antipodal Vivaldi antenna as a function of frequency.

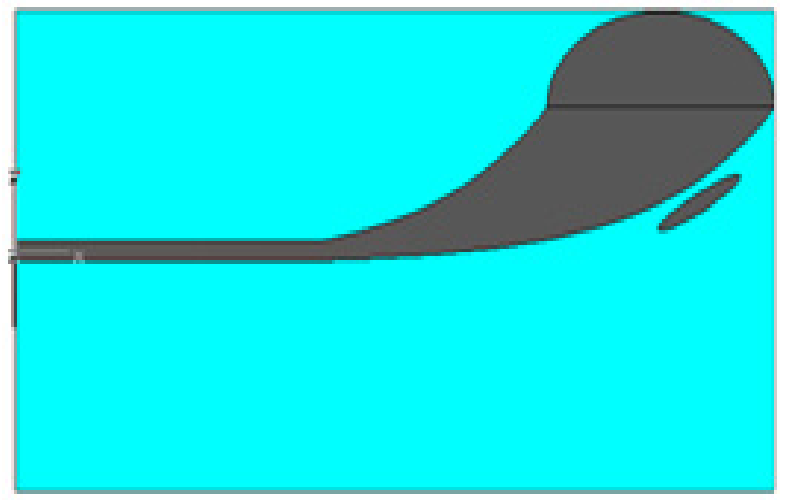

Fig. 3. Top view of modified AntipodalVivaldi antenna.
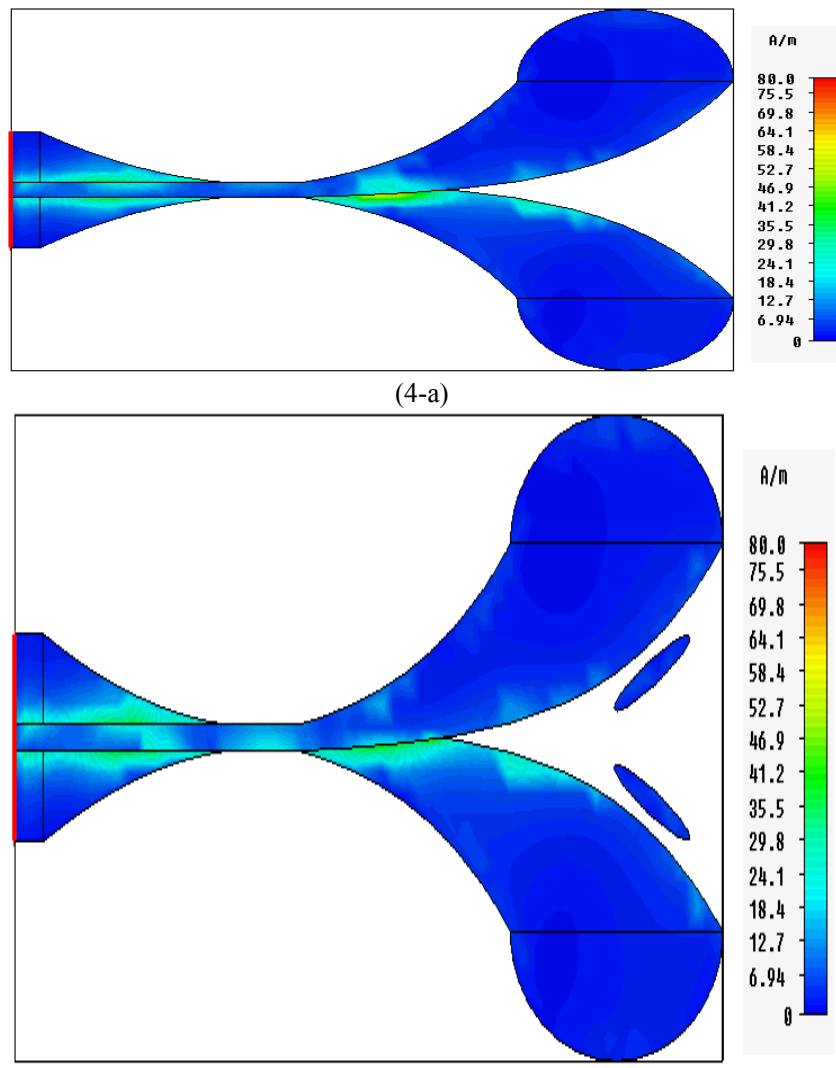

(4-b)

Fig. 4. Surface current distribution at $10.5 \mathrm{GHz}$ a) common antenna b) modified antenna.

The color ranges in Fig. 4-a and 4-b are chosen in the same range $(0-80 \mathrm{~A} / \mathrm{m})$ so that the surface currents can be compared. As it is shown, the current tends to distribute smoother in the presence of the parasitic element (Fig. 4-b), especially in the transition area between transmission line and the radiator part.

Parameters of the elliptical strip are the radius of length and width. In an optimization process, done by simulation tools, these two parameters, also the position of the strip on the substrate, were optimized for minimum $\left|S_{11}\right|$ in the frequency range. They are obtained as $a=3 \mathrm{~mm}, b=0.5 \mathrm{~mm}$, $\mathrm{x}=45 \mathrm{~mm}, \mathrm{y}=2.5 \mathrm{~mm}$ where $\mathrm{a}$ is radius of length, $\mathrm{b}$ is radius of width, $\mathrm{x}$ is the $\mathrm{x}$ axis position and $\mathrm{y}$ is the $\mathrm{y}$ axis position.

For comparison, in Fig. 5 the results for the common and the modified antenna are presented. As states before for $\left|\mathrm{S}_{11}\right|<-10 \mathrm{~dB}$, the lower frequency band of the common antenna is $9.13 \mathrm{GHz}$, while it is $8.22 \mathrm{GHz}$ for the modified one, hence an increase of about $0.9 \mathrm{GHz}$ for the lower 
frequency band.

Despite the fact that $\left|S_{11}\right|$ variations improves in the modified structure, the antenna gain does not change significantly in the frequency range, as can be seen in Fig. 5-b.

Fig. 6 presents the radiation patterns ( $\mathrm{E} \& \mathrm{H}$ plane) of both common and the modified structures at 10 and $15 \mathrm{GHz}$.
It can be seen that there is no remarkable difference between the radiation patters of these two structures. In fact, in the modified antenna the beamwidth is less than that of the common structure (about 2 degrees) since the gain in these frequencies $(10 \& 15 \mathrm{GHz})$ is slightly higher in the modified Antipodal Vivaldi antenna.
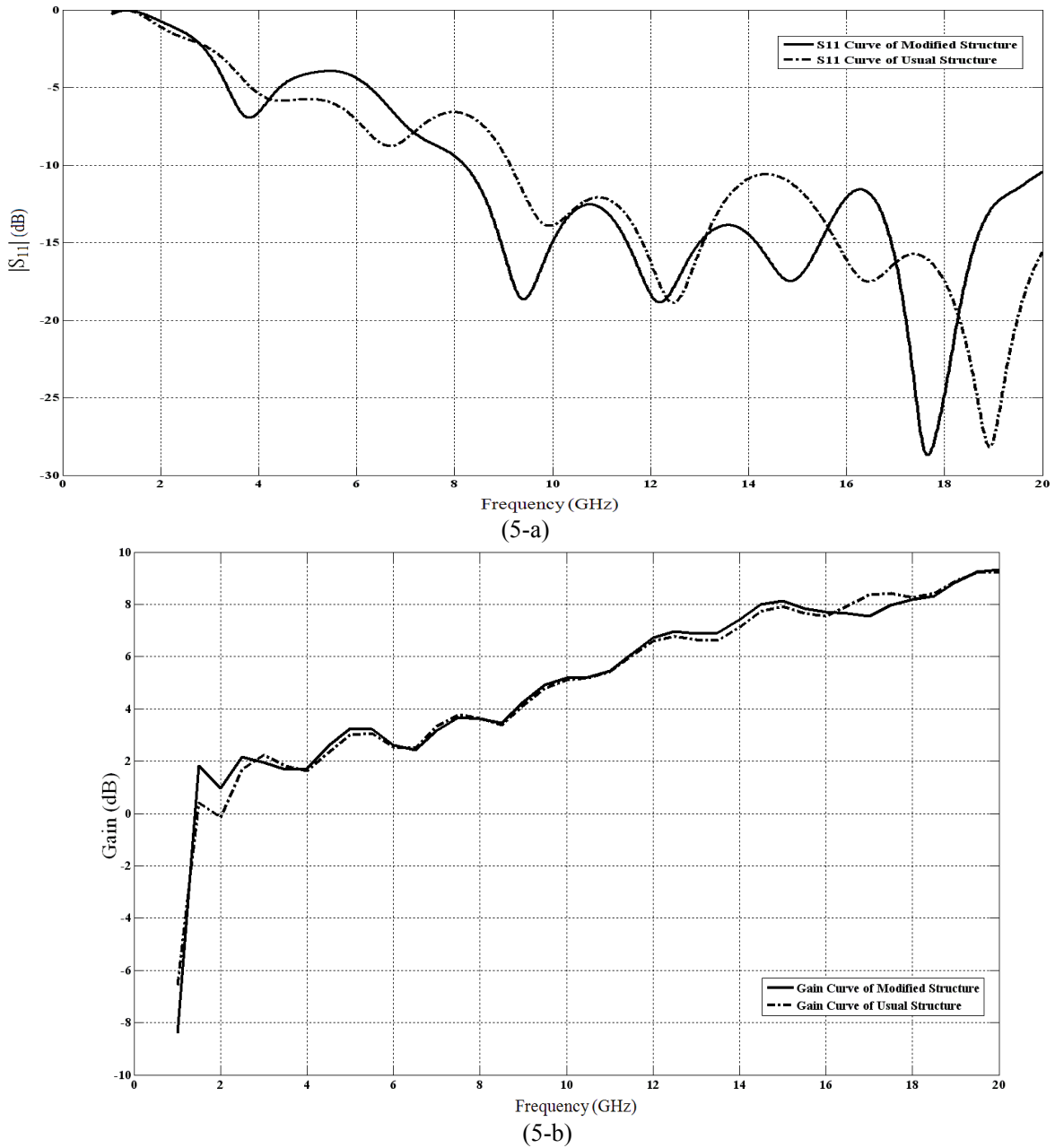

Fig. 5. Comparison between common and modified Antipodal Vivaldi antenna a) $\left|S_{11}\right|$ b) Gain, versus frequency.
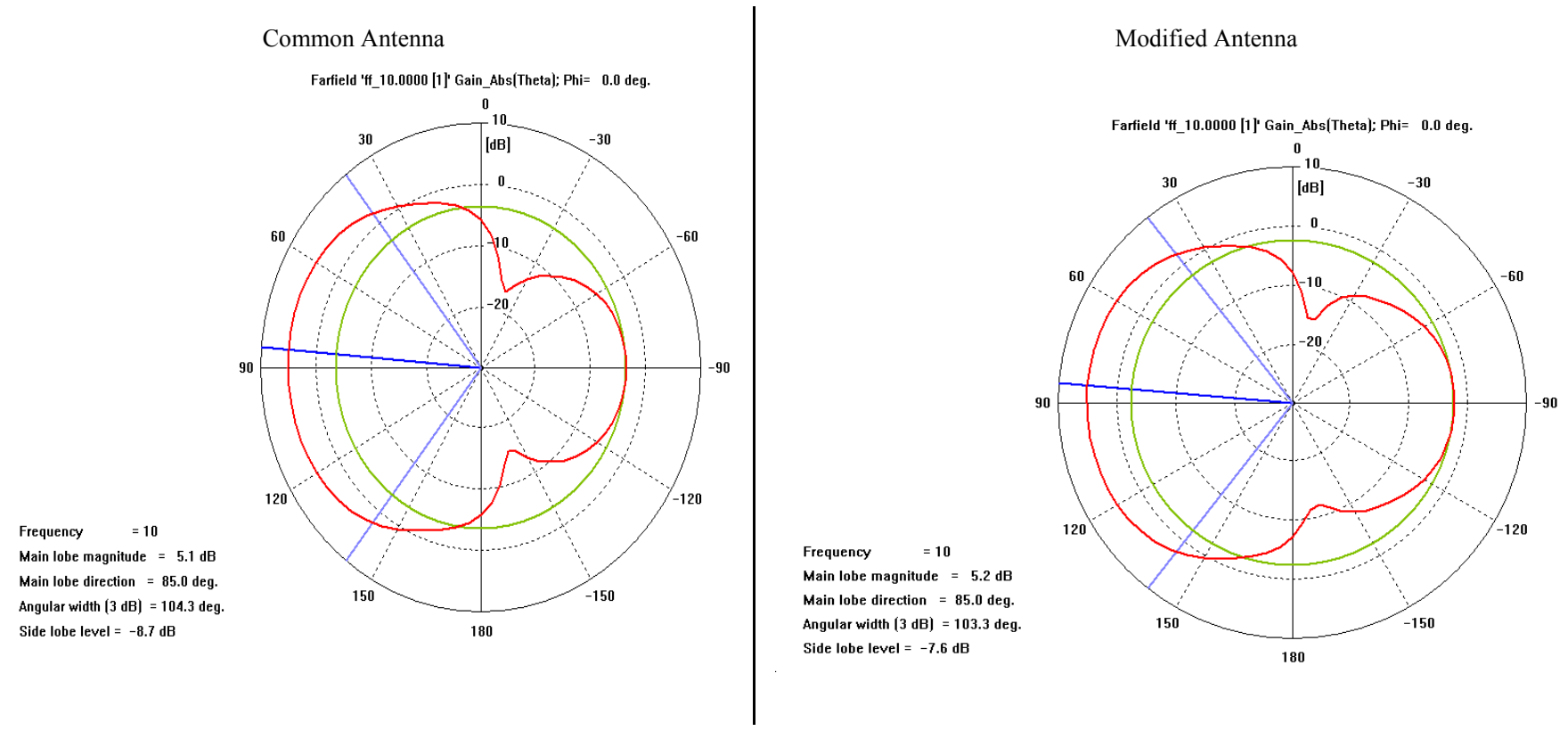


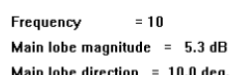
Main lobe direction $=10.0 \mathrm{deg}$ Angular width $(3 \mathrm{~dB})=81.1 \mathrm{deg}$ Side lobe level $=-2.7 \mathrm{~dB}$

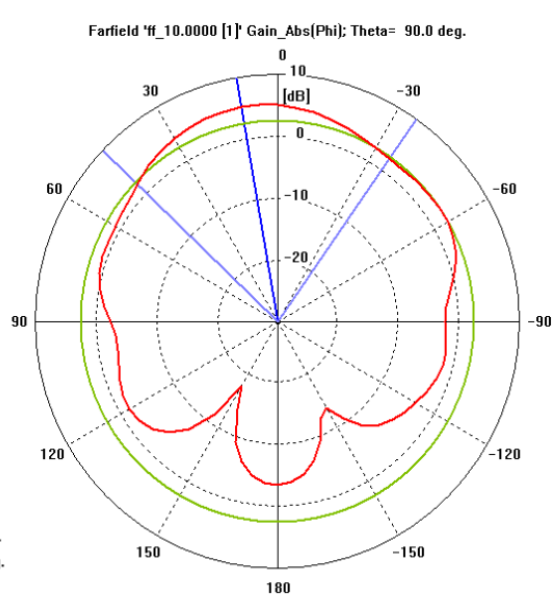

Common Antenna

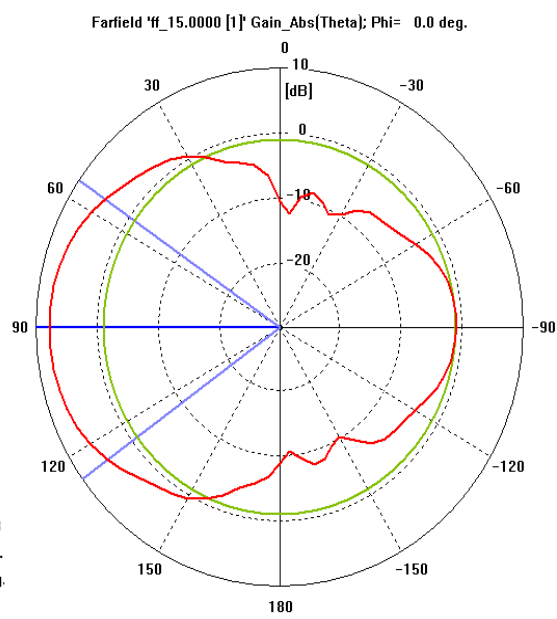

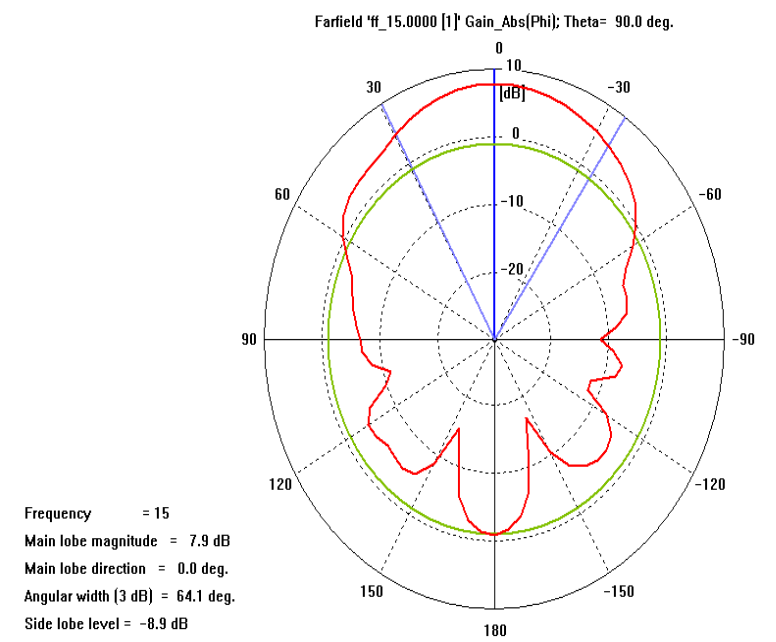

Frequency Main lobe direction $=10.0 \mathrm{deg}$ Angular width $(3 \mathrm{~dB})=78.2$ deg. Side lobe level $=-3.3 \mathrm{~dB}$

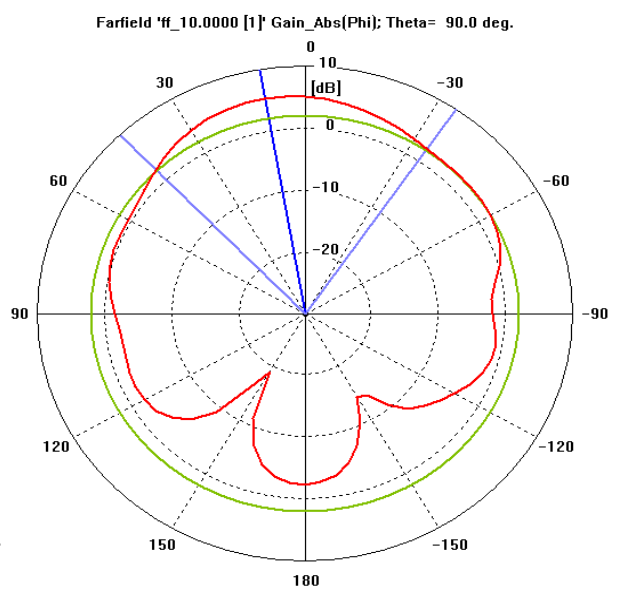

(6-a)
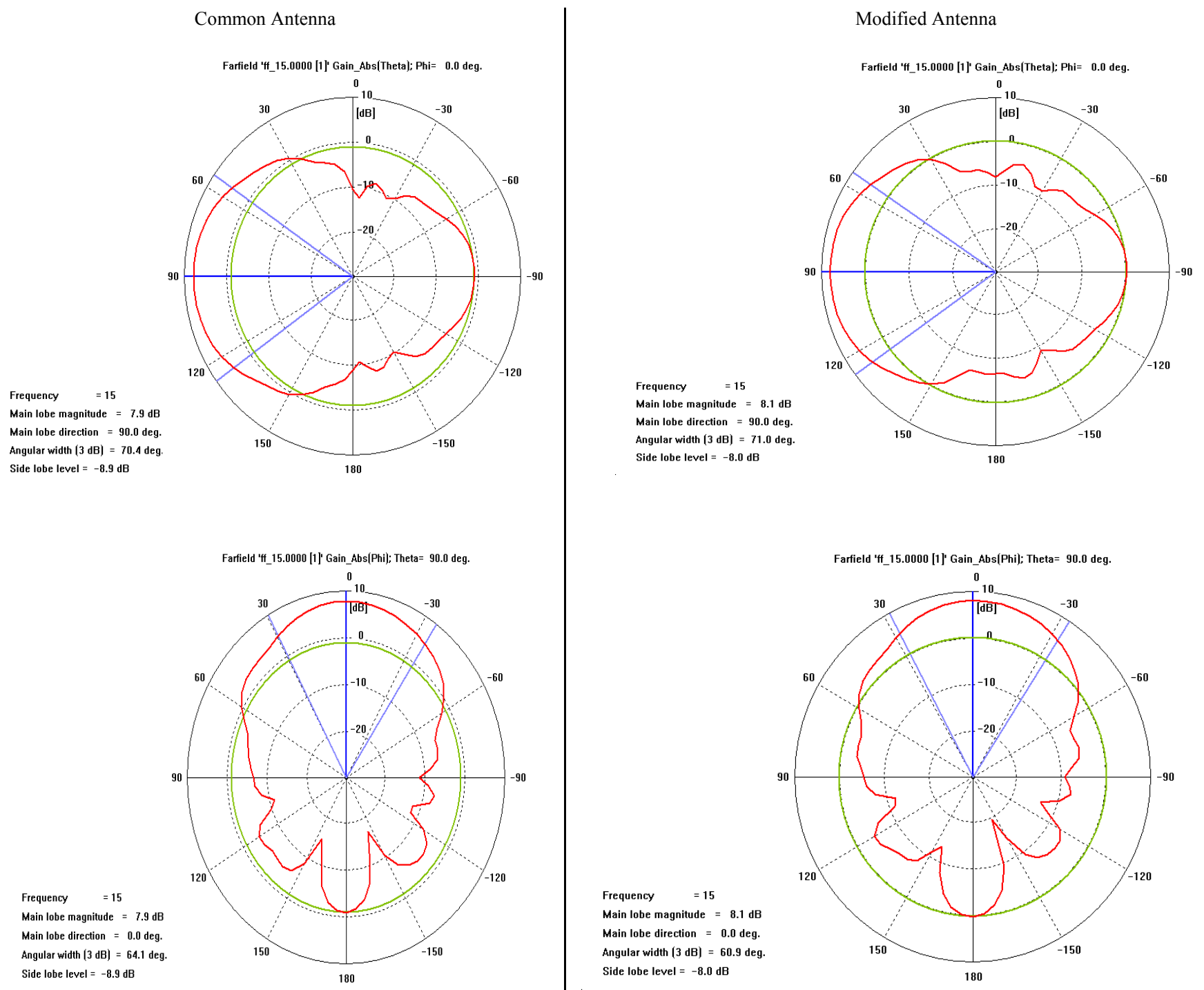

Main lobe magnitude $=8.1 \mathrm{~dB}$ Main lobe direction $=\mathbf{9 0 . 0}$ deg Angular width $(3 \mathrm{~dB})=71.0 \mathrm{deg}$ Side lobe level $=-8.0 \mathrm{~dB}$

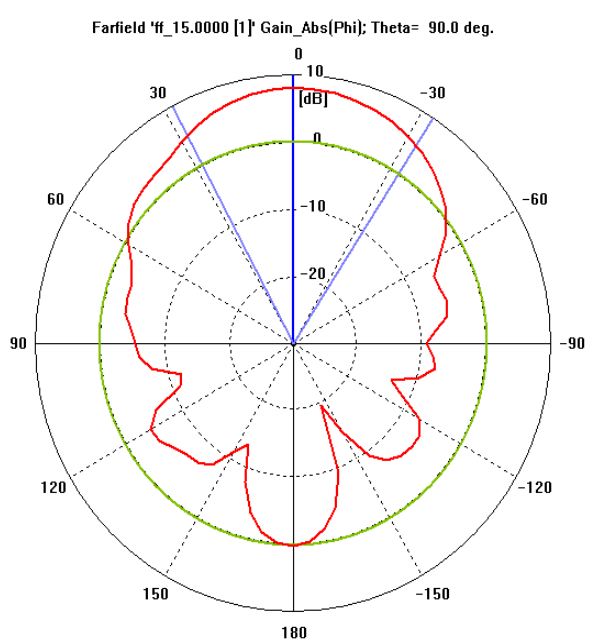

(6-b)

Fig. 6. Comparison of $\mathrm{E} \& \mathrm{H}$ patterns of common and modified Antipodal Vivaldi antenna a) at $10 \mathrm{GHz}$ b) at $15 \mathrm{GHz}$.

\section{CONCLUSIONS}

The effects of inserting a parasitic element into the common Antipodal Vivaldi antenna on the input impedance and radiation gain have been investigated. All simulations were carried out by CST Microwave Studio simulation tools. The following results have been achieved: 
Adding an elliptical strip on the substrate can decrease the lower frequency band while the variation of $|S 11|$ remains smaller than $-10 \mathrm{~dB}$ in the rest of the band.

With improvement in $\left|S_{11}\right|$ of the modified structure, the antenna gain manifests no significant changes.

The radiation patterns do not remarkably differ in these two structures.

It is clear that the addition of the parasitic element does not change the cost and the complexity of the antenna manufacture.

\section{REFERENCES}

[1] S. Wang, X. D. Chen and C. G. Parini, "Analysis of Ultra Wideband Antipodal Vivaldi Antenna Design," IEEE Loughborough Antennas and Propagation Conference, 2-3 April 2007, Loughborough, UK.

[2] E. Gazit, "Improved design of the Vivaldi antenna," IEE Proceedings, Vol.135, pp. 89-92, April 1988.

[3] D.H. Schaubert et al, "Wide bandwidth Vivaldi antenna arrays - some recent developments," Proceedings of 'EuCAP 2006', 6-10 November 2006, Nice France.

[4] J. J. Lee, "Slotline impedance", IEEE Trans. on MTT, Vol. 39, pp. 666-672, April 1991. 